\title{
Psychotherapie im Kontext von Differenz, (Macht-)Ungleich- heit und globaler Verantwortung
}

\author{
Diversity \& Intersectionality als hilfreiche Perspektiven für eine \\ gesellschaftskritische Psychotherapie
}

\author{
Leonore Lerch
}

Online publiziert: 17. Mai 2019

(C) Der/die Autor(en) 2019

Zusammenfassung Der Artikel gibt eine Einführung in die Ansätze von diversity \& intersectionality und beleuchtet diese unter einer interaktionstheoretischen sozialkonstruktivistischen Perspektive (doing difference, doing intersectionality). Intersektional orientierte Konzepte finden vor allem bei der Analyse von Ungleichheit und Diskriminierung Anwendung. Eine Grundlage für Diskriminierung bilden Prozesse des othering, die im Kontext von Machtdiskursen der Herstellung von Dominanz dienen. Wie das Modell des minority stress nachweist, führen fortdauernde Erfahrungen von Diskriminierung und struktureller Unterdrückung insbesondere für Angehörige von Minderheiten zu einer erhöhten Stressbelastung, die mit negativen Folgen für die psychische Gesundheit verbunden sind. In Summe können häufige Erfahrungen von Mikroaggressionen im Zusammenhang mit Diskriminierung zu kumulativen bzw. sequentiellen Traumatisierungen führen. Abschließend bietet der Artikel Empfehlungen an die Psychotherapie zur Entwicklung einer differenz- und dominanzkritischen psychotherapeutischen Haltung und fordert unter Bezugnahme auf die Menschenrechte einen Perspektivenwechsel hin zu einer globalen Verantwortung.

Schlüsselwörter Diversity · Intersectionality · Diskriminierung · Othering · Mikroaggression • Minderheitenstress · Trauma - Psychotherapie als Menschenrechtsprofession · Globale Verantwortung

L. Lerch $(\bowtie)$

Herminengasse 4/11, 1020 Wien, Österreich praxis@leonore-lerch.at
Psychotherapy in the context of difference, (power)inequality and global responsibility Diversity \& Intersectionality as helpful perspectives for sociocritical psychotherapy

Summary The article gives an introduction to the approaches of diversity \& intersectionality and illuminates them under an interaction-theoretical social constructivist perspective (doing difference, doing intersectionality). Intersectional oriented concepts are mainly used in the analysis of inequality and discrimination. A basis for discrimination is formed by processes of othering that serve to establish dominance in the context of power discourses. As the model of minority stress demonstrates, continuing experiences of discrimination and structural repression, especially for members of minorities, result in increased levels of stress associated with negative consequences for mental health.

In sum, frequent experiences of microaggression related to discrimination can lead to cumulative or sequential trauma. Finally, the article offers recommendations to psychotherapy for the development of a difference-critical and dominance-critical psychotherapeutic attitude and calls for a change of perspective towards global responsibility with reference to human rights.

Keywords Diversity - Intersectionality • Discrimination · Othering · Microaggression • Minority stress · Trauma · Psychotherapy as a human rights profession · Global responsibility

\section{Einleitung}

Seit den 90er Jahren werden im deutschsprachigen Raum Debatten um gender teilweise von diversity 
abgelöst. Diversity thematisiert nicht nur die gender-Differenz, sondern weitere Differenzlinien wie Herkunft, Ethnizität, race, Hautfarbe, sozialer Status, Klasse, Besitz, Gesundheit, Sexualität etc. und kann als Erweiterung der gender-Perspektive betrachtet werden. Differenzen entstehen nicht zufällig oder beliebig, „sondern in einer historisch aufklärbaren Weise regelmäßig [...] entlang bestimmter Differenzverhältnisse“ (Mecheril und Plößer 2018, S. 287). Analog zum Ansatz des doing gender (West und Zimmerman 1987) etablieren West und Fenstermaker (1995) das Konzept des doing difference „als einem Prozess, durch den Individuen sich durch Rückgriff auf unterschiedliche Kategorien wie ,Kultur ${ }^{\prime}$ oder ,Geschlecht' darstellen bzw. entlang dieser Kategorien Zuschreibungen erfahren“ (Mecheril und Plößer 2018, S. 286). Die doing-Ansätze entstammen ursprünglich der interaktionstheoretischen Soziologie und gehen von einem sozialkonstruktivistischen Verständnis aus, nach dem psychische, soziale und gesellschaftliche Phänomene nicht natürlich bzw. essentialistisch festgeschrieben existieren, sondern durch Handeln performativ hergestellt werden, was ihre Prozesshaftigkeit, Veränderlichkeit und Kontextualität hervorhebt. Diversity findet Anwendung in Gleichstellungsund Antidiskriminierungspolitiken sowie in Debatten um Menschenrechte. Privatwirtschaftliche Unternehmen setzen Konzepte von diversity management bzw. managing diversity im Rahmen von Werbekonzepten oder der Produktentwicklung ein und versuchen, die Unterschiede zwischen Mitarbeiter_innen oder Kund_innen als Wettbewerbsvorteil zu nutzen. Hier liegt auch die Kritik an diesen diversity-Ansätzen begründet, da Fragen der Chancengleichheit oder Gerechtigkeit in den Hintergrund rücken und Machtverhältnisse eher ausgeblendet werden (Walgenbach et al. 2012). „Diversity without changing the structure, without calling for structural formation, simply brings those who were previously excluded into a process that continues to be as racist, as misogynist as it was before" (Davis 2018, zit. n. Gonzalez 2018, o. S.).

In der Psychotherapie wird diversity meist hinsichtlich differenter Klient_innengruppen assoziiert. Wir arbeiten mit Gesundheits- und Krankheitsbegriffen, die sich schulenspezifisch unterscheiden. Psychiatrische Diagnosen beschreiben Konstrukte der Abweichungen von psychischer Normalität. Wir bewegen uns in einem permanenten Spannungsfeld zwischen „der Reproduktion von Normalitätsmustern und Andersheit“ (Mecheril und Plößer 2018, S. 284). Psychotherapie ist folglich einerseits ein Normalisierungsinstrument (Butler 2009, S. 366), also ein Instrument, um Normalität herzustellen, kann aber in emanzipatorischem Sinne gleichzeitig dazu beitragen, Normalität in Frage zu stellen und zu dekonstruieren.

Im deutschsprachigen Raum forderte bereits die feministische Psychotherapie der 70er und 80er Jahre die Reflexion der gender-Differenz in der Psychotherapie. Scheffler (2010) bietet einen historischen
Überblick zu den Entwicklungen und differenten Positionierungen der feministischen Psychotherapie aus Weißer ${ }^{1}$ Perspektive. Die Kongressdokumentation des 22. Feministischen Frauentherapiekongresses (Hahn et al. 1999) ist eines der wenigen historischen Zeitdokumente, das Schwarze ${ }^{2}$ Positionierungen zur feministischen Psychotherapie und Beratung für den deutschsprachigen Raum liefert. Die Globalisierung und der damit einhergehende demographische Wandel, wirtschaftliche Not, Krieg, Flucht- und Migrationsbewegungen führen $\mathrm{zu}$ weiteren Differenzen hinsichtlich Herkunft, Nationalität, Sprachen, Religionen und kultureller Prägungen. Vor allem für die Arbeit mit Klient_innen aus sozialen Gruppen, die nicht der Mehrheitsgesellschaft bzw. Dominanzkultur (Rommelspacher 1995) angehören z. B. Menschen mit lesbian-gay-bi-transgender-intersexual- *queer-Hintergrund (LGBTI*Q), mit Flucht- und/oder Migrationsbiographien, mit Handicaps, Kinder, alte Menschen, Frauen - erweist sich diversity als hilfreiches Konzept, da es die Auseinandersetzung mit pluralistischen Lebensformen fördert und eine Sensibilisierung für die Bedürfnisse und Problemlagen unterschiedlicher sozialer Gruppen ermöglicht.

\section{Diversität und Intersektionalität}

Intersectionality geht insofern über diversity hinaus, als es die Mehrdimensionalität und Verwobenheit von Zugehörigkeiten oder Zuschreibungen zu bestimmten Diversitätskategorien betrachtet und soziale Kategorien nicht „durch eine einfache Addition der Differenzlinien (,Geschlecht' plus ,Klasse“ plus ,Behinderung')“ fasst (Mecheril und Plößer 2018, S. 287). Dabei steht die Analyse von Unterdrückungs- und Diskriminierungsprozessen im Kontext von Mehrfachzugehörigkeiten im Vordergrund. Intersektionalität kann auch als Weiterentwicklung von gender-Ansätzen betrachtet werden und verweist ebenso wie gender auf eine herrschaftskritische Tradition, die „Fragen globaler Gerechtigkeit durch Umverteilung“ ins Zentrum stellt (Smykalla und Vinz 2011, S. 11).

Der Begriff intersectionality wurde 1989 von der Schwarzen US-amerikanischen Rechtswissenschaftlerin Kimberlé Williams Crenshaw geprägt. Sie entwickelte anhand ihrer Analyse von Gerichtsentscheidungen $\mathrm{zu}$ mehrdimensionalen Diskriminierungsfällen das Bild einer Kreuzung (intersection) und „kritisiert damit einen ,single-axisapproach', also einen auf separate Gründe oder Merkmale zielenden Ansatz von

\footnotetext{
1 Die Großschreibung von Schwarz ist eine politische Selbstbezeichnung und Selbstpositionierung und beschreibt eine von Rassismus betroffene gesellschaftliche Position. Schwarz wird verstanden als eine politische Identität, nicht als biologisches Merkmal oder Zuschreibung.

2 Die Großschreibung von Weiß bezeichnet eine nicht von Rassismus betroffene gesellschaftliche Macht-Position und nicht ein biologisches Merkmal.
} 
Antidiskriminierungspolitik, als unzureichend und hinderlich für die Beseitigung von Diskriminierung. Er nehme Diskriminierte als entweder rassistisch oder sexistisch oder aus anderen Gründen diskriminiert wahr, begreife aber spezifische, eben intersektionale Diskriminierungen nicht“ (Baer et al. 2010, S. 10-11).

McCall (2001) unterscheidet drei methodologische Zugänge $\mathrm{zu}$ intersectionality. Zum einen werden die Wechselwirkungen innerhalb einer Kategorie analysiert (intrakategorialer Ansatz) - beispielsweise die Frage, wie sich ,Geschlecht' in Verschränkung mit ,Klasse‘ und ,körperlicher Befähigung‘ ausformt. Zum anderen stehen die Überkreuzungen zwischen den Kategorien im Fokus (interkategorialer Ansatz) - also die Interdependenzen, in denen sich z.B. die Kategorien ,Geschlecht', ,Klasse' und ,körperliche Befähigung' zueinander befinden sowie ihre gegenseitige Beeinflussung. Eine dritte Perspektive von intersectionality (antikategorialer Ansatz) nimmt nicht Bezug auf die sozialen Kategorien, sondern die Prozesse und Strukturen, die zu Privilegierungs- oder Diskriminierungsdynamiken führen (vgl. Hofmann 2017). Degele und Winker (2009) untersuchen in ihrem Mehrebenenmodell zudem drei Ebenen, auf denen sich die Verwobenheit und Durchdringung der Differenzlinien abzeichnen. Sie beschreiben eine Identitätsebene (Ebene der Identitätskonstruktionen), Repräsentationsebene (Ebene der symbolischen Repräsentationen z.B. Wissen, Werte, Menschenbilder etc.) und Strukturebene (Ebene gesellschaftlicher Systeme z. B. Gesundheitswesen, Psychotherapiegesetz). Kerchner (2011) erforscht Intersektionalität anhand diskursanalytischer Perspektiven, die sie auf Fragestellungen im Kontext von Differenz- und Diskriminierungserfahrungen und der Konstruktion von Wir und die Anderen anwendet. Wie bei diversity beziehen intersectionalityAnsätze eine unterschiedliche Anzahl von Differenzkategorien mit ein. Beispielsweise gehen Klinger und Knapp (2007) von den drei Kategorien race, class, gender aus, Degele und Winker ergänzen als vierte Kategorie ,Körper‘. Statt generell die Anzahl der Kategorien auszuweiten, um eine Hierarchisierung der Differenzlinien zu vermeiden, plädieren Smykalla und Vinz (2011) dafür, den Fokus auf die Relevanz einzelner Kategorien für das jeweilige Forschungsinteresse zu legen und kontextabhängig zu bewerten.

\section{Historische Wurzeln}

Hervorgegangen sind die Diskurse um Diversität und Intersektionalität aus den internationalen Frauenbewegungen und Schwarzen Bürgerrechtsbewegungen in den USA der 70er Jahre. Schwarze Frauen und women of color kritisierten, dass sich die feministischen Theorien und politischen Forderungen nur an den Interessen Weißer, heterosexueller Feministinnen aus der Mittelschicht orientierten. Sie vertraten die Ansicht, dass Schwarze Frauen in den Systemen von race und class im Verhältnis zu Weißen Frauen unterschiedliche Positionen einnehmen und dementsprechend unterschiedliche Erfahrungen machten. Im Kontext der critical race theory sind zahlreiche intersektionale Ansätze entstanden u.a. Combahee River Collective (1981), hooks (1981), Hull et al. (1982), Davis (1982), Lorde (1988), Collins (1998) (vgl. Baer et al. 2010).

Bereits 1851 stellte die Schwarze Frauenrechtlerin Sojourner Truth eine Verbindung zwischen Sexismus und Rassismus her und kritisierte sowohl die Frauen- als auch Sklav_innenrechte. Legendär wurde ihre Rede auf einer Frauenrechtskonvention in Akron, Ohio, auf der sie die prominent gewordene Frage „Ain't I a woman? ${ }^{\circ}$ an die anwesenden Weißen Männer richtete, die Frauen aufgrund ihrer angeblichen Minderwertigkeit kein Stimmrecht zuerkannten. Sojourner Truth thematisierte aber nicht nur den Sexismus Weißer privilegierter Männer, sondern auch den Rassismus und die Klassenherrschaft in den Weiß dominierten Frauenbewegungen (vgl. Davis 1982).

Auch im deutschsprachigen Raum waren es vor allem Schwarze Frauen, Frauen mit Migrationsbiographien, lesbische Frauen, jüdische Frauen, Frauen mit Behinderung, die seit den 80er Jahren auf die Mehrdimensionalität von Ungleichheitsverhältnissen hinwiesen (vgl. Lerch 1997a). Der feministische Slogan „Das Private ist politisch!“, der den Dualismus von Privatheit und Öffentlichkeit in Frage stellt und die gesellschaftliche Bedingtheit sozialer Probleme aufzeigt, wurde als Perspektive Weißer Mittelschichtsfrauen dekonstruiert. Die Erfahrung der Getrenntheit von privatem und öffentlichem Raum basiert bereits auf einer Privilegierung, über die Schwarze Menschen historisch nicht verfügten, da gesellschaftliche Institutionen ständig in ihre Familien und Gemeinschaften eingriffen (vgl. Klaus 2005). Die persönliche Privatheit vor einer Öffentlichkeit schützen zu können, setzt bereits ein gewisses Maß an gesellschaftlicher Macht voraus. Schwarze Frauen begründeten ihren Zugang zum Persönlichen in einer Politik der Gruppenstandpunkte und nicht auf einem Modell, das auf dem Eintreten für individuelle Interessen basiert. „Ein Zeichen der Weißen Privilegiertheit besteht ironischerweise in der Fähigkeit, sich selbst als Individuum zu betrachten und darüber hinwegzusehen, wie die bloße Mitgliedschaft in der Weißen Gruppe das Privileg der Individualität hervorbringt. [...] Politische Strategien, die davon ausgehen, dass die grundlegende Einheit einer politischen Bewegung eine Person ist, die bürgerliche Rechte besitzt, werden erst im Zusammenhang

\footnotetext{
3 „Ain’t I a woman?“. Auszug aus der Rede von Sojourner Truth (1851): „Look at me! Look at my arm [... I I have ploughed, and planted, and gathered into barns and no man could head me! And ain't I a woman? I could work as much and eat as much as a man - when I could get it - and bear the lash as well! And ain't I a woman? I have borne thirteen children and seen them most all sold off to slavery, and when I cried out with my mother's grief, none but Jesus heard me! And ain't I a woman?“ (Truth 1851, zit. n. Davis 1982, S. 62).
} 
mit Rassenprivilegierung und der damit verbundenen Vorstellung von Individualität verständlich“ (Collins 1996, S. 86).

\section{Intersektionale Perspektiven auf Diskriminierung und Identität}

Diskriminierung wird hergestellt durch die gesellschaftsbedingte Hierarchisierung und Ungleichgewichtung von binär gefassten Differenzkategorien (männlich-weiblich, heterosexuell-homosexuell, gesund-krank, Weiß-Schwarz, inländisch-ausländisch, reich-arm etc.) und ist häufig mehrdimensional, weil mehrere miteinander verwobene Differenzlinien betroffen sind. Insofern kann auch von einem doing intersectionality gesprochen werden, denn die hierarchisierten gesellschaftlichen Verortungen, entlang derer sich Menschen bewegen, werden durch soziales Handeln hergestellt, ,allerdings sind die Handlungsspielräume der Subjekte nicht unbegrenzt und frei, sondern vorstrukturiert und in normative Ordnungen eingelassen“ (Mecheril und Plößer 2018, S. 286). Wie die Ontario Human Rights Commission 1997 anhand von Beschwerden wegen Diskriminierung feststellte, stehen in $48 \%$ der Fälle die Diskriminierungsgründe in Verbindung mit mehr als einem, der Persönlichkeit zugeschriebenen Merkmal (vgl. Gummich 2004). Menschen erfahren nicht entweder ihre geschlechtliche oder sexuelle oder ethnische Identität als voneinander getrennte Aspekte, sondern die jeweiligen situationsund kontextabhängigen Verflechtungen dieser Differenzlinien bringen unterschiedliche Erfahrungen von Identität hervor. Diskriminierungserfahrungen wirken in Identitätsprozesse hinein und (ver-)formen diese, weil die gesellschaftlichen Zuschreibungen an den Identitätskonstruktionen anknüpfen und sie in Frage stellen, verzerren oder verletzen können. „Die Erkenntnis, dass intersektionelle Diskriminierung existiert, geht mit der Erkenntnis einher, dass es eine ,intersektionelle Identität' gibt“ (Gummich 2004, S. 8).

Identität beschreibt fluide Prozesse der Identifikation mit persönlichen Merkmalen und sozialen Gruppen im Kontext normativer sozialer und gesellschaftlicher Machtverhältnisse. Keupp und Bilden (1989) prägen den Begriff der multidimensionalen patchwork-Identität, deren Ziel nicht ist, Differenzen und Widersprüche aufzulösen, sondern vielmehr die verschiedenen Aspekte von Identität in ein stimmiges Spannungsverhältnis zueinander $\mathrm{zu}$ bringen. Lorde (1994/1982) spricht von many selves oder house of difference. Identität ist nicht etwas, das wir haben oder das wir sind. Identität wird in Prozessen von doing identity erfahrbar (vgl. Butler 2002). Hall (1994) geht unter Bezugnahme auf Foucault von einem diskursiven Verständnis von Identität aus, das Identität nicht als Einheit im Zentrum von sozialen Ordnungen betrachtet, sondern sich denken lässt als inkonsistente Verdichtungspunkte in einem losen Netzwerk, die erst durch die Diskurse selbst hervorgebracht und wieder verflüchtigt werden. Eine Folge der Dezentrierung und Dekonstruktion des Subjektes der Postmoderne sind, inmitten von immer komplexer werdenden Lebenszusammenhängen, Erfahrungen zunehmender Fragmentierung, Unbeständigkeit, Widersprüchlichkeit und Verunsicherung.

Freude aus Verunsicherung ziehn - wer hat uns das je beigebracht! (Christa Wolf 1983, S. 131)

\section{Othering als Prozesse der Herstellung von Normalität und Dominanz}

Im postkolonialen Diskurs bezeichnet othering, wie „das im Machtdiskurs ausgeschlossene Andere“ kreiert wird (Spivak 1985, S. 255). Durch die sozialpsychologischen Mechanismen des othering - verstanden als einem doing otherness - werden Subjekte als die Anderen, die Fremden konstruiert, klassifiziert und sichtbar gemacht, während die eigenen Subjekt-Positionen unberührt und unmarkiert bleiben (vgl. Lerch 2011). Ziel ist die Herstellung von Dominanz, indem das eigene soziale Image hervorgehoben und aufgewertet, den Anderen jedoch eine inferiore Position zugewiesen wird. Othering bildet eine Grundlage für Diskriminierung und stellt widersprüchliche Dynamiken von Identifikation und Internalisierung her, da Positionen für Handlungsfähigkeit oder Widerständigkeit nur innerhalb von Machtbeziehungen markiert werden. „Wo es Macht gibt, gibt es Widerstand. Und doch oder gerade deswegen liegt der Widerstand niemals außerhalb der Macht. [... ] Die Widerstände rühren nicht von irgendwelchen ganz anderen Prinzipien her. [...] Sie sind in den Machtbeziehungen die andere Seite, das nicht wegzudenkende Gegenüber“ (Foucault 1983, S. 116f). Die Identifikation mit den hegemonialen Normen, und die Anpassung an sie, gehen zwar einher mit der Erfahrung der Unterwerfung, ermöglichen jedoch eine minorisierte Subjektivität. „Das Subjekt lässt sich durchaus so denken, dass es seine Handlungsfähigkeit von eben der Macht bezieht, gegen die es sich stellt" (Butler 2001, S. 22). Internalisierungs- und Identifikationsprozesse können als Bewältigungsstrategien verstanden werden, die das Überleben und die Handlungsfähigkeit von Subjekten sichern sollen, jedoch häufig einhergehen mit einem Verlust von Selbstwert, Integrität und Autonomie (vgl. Böhnisch 2016). Werden die stereotypen Differenzordnungen durch die Anderen nicht bestätigt, reagieren Angehörige der Dominanzgesellschaft nicht selten mit Irritation und Mikroaggressionen (Carter 2007). „Woher kommen Sie? Aus Wien. Nein, ich meinte, woher kommen Sie ursprünglich? Aus Wien. Nein, Sie verstehen nicht. Ich meinte, wo sind Sie geboren? In Wien. Tatsächlich? Aber Sie sehen gar nicht so aus!" 


\section{Diskriminierung und Trauma}

Die Auswirkungen von individueller oder struktureller Diskriminierung auf die Gesundheit sind anhand der Forschung mit LGBTI*Q-Personen ${ }^{4}$ gut belegt (vgl. Mahler et al. 2018). Wie das Modell des minority stress von Meyer (2003) zeigt, erleben minorisierte Gruppen den Stress nicht nur als vorübergehende, sondern als permanente Belastung, die sich anhaltend negativ auf die psychische Gesundheit auswirken kann.

$\mathrm{Zu}$ rassistischer Diskriminierung und ihren Folgen für die psychische Gesundheit wurde im deutschsprachigen Raum bislang noch kaum geforscht. Igel et al. (2010) stellen in einer repräsentativen Haushaltsbefragung von 1844 Migrant_innen in Deutschland den negativen Einfluss von Diskriminierungserfahrungen auf die Gesundheit fest. Der Fokus bei deutschsprachigen Untersuchungen liegt jedoch mehr auf den Stressfaktoren infolge von Migrationsprozessen und Erfahrungen kultureller Differenz und weniger auf den Ungleichheits- und Diskriminierungserfahrungen. Im Vergleich dazu sind die Ergebnisse der internationalen Forschung in den USA, Großbritannien, Australien, Neuseeland und Südafrika eindeutig. Rassismus stellt einen wesentlichen Bestimmungsfaktor für die psychische Gesundheit von Minderheitenangehörigen dar. Eine Stichproben-Untersuchung der indigenen Bevölkerung Australiens von Paradies \& Cunningham (2012) „identifizierte mehrere Einflussfaktoren, die die Entwicklung einer Depression in der Zielperson rassistischer Diskriminierung unterstützten: fehlende Kontrolle über das Ereignis, Stress, negative soziale Kontakte, sowie Gefühle von Scham und Ohnmacht. Diese Einflussfaktoren zusammen waren für $66 \%$ der Assoziationen zwischen interpersonellem Rassismus und depressiven Symptomen verantwortlich" (Yeboah 2015, S. 12). Weitere Untersuchungen sind von Loo et al. (2001), Comas-Días \& Jacobsen (2001) und Butts (2002) belegt. Die Analyse von Bryant-Davis \& Ocampo (2005a, 2005b) markiert wichtige Parallelen zwischen Rassismus, Vergewaltigung und häuslicher Gewalt (vgl. ebd.).

Rassistische Diskriminierung wird oft nicht „als Trauma wahrgenommen und benannt. Diese Absenz der Benennung liegt daran, dass die Geschichte der rassistischen Unterdrückung und ihre psychologische Auswirkung innerhalb des westlichen Diskurses bisher vernachlässigt wurde. Schwarze Menschen und people of color sind damit jedoch tagtäglich konfrontiert. Sie müssen nicht nur auf einer individuellen Ebene, sondern auch auf einer historischen und kollektiven Ebene mit den Traumata der Sklaverei und des Kolonialismus sowie dem Gefühl der Scham umgehen“ (Kilomba 2008, S. 133). Carter (2007) entwickelte anhand seiner Untersuchungen zu Rassismusbedingten Belastungen das Modell des race-based

\footnotetext{
${ }^{4}$ lesbian-gay-bi-transgender-intersexual- ${ }^{*}$ queer-Personen.
}

traumatic stress. Dabei müssen die belastenden Erfahrungen z.B. alltägliche rassistische Mikroaggressionen in Form von non-/verbalen Beleidigungen, Kränkungen, Demütigungen etc. als Einzelereignisse nicht traumatisierend wirken. In Summe jedoch können sie zu einer traumatisierenden Gesamtbelastung führen. Khan (1963) spricht in diesem Zusammenhang von einem kumulativen Trauma. Keilson (1979) kommt in seinem Konzept der sequentiellen Traumatisierung zum Ergebnis, dass mehrfache Erfahrungen von traumatisierenden Ereignissen zu einer Traumaverschärfung und, nach dem Ende der unmittelbaren Belastungssituation, zu anhaltenden Stressreaktionen führen können. Insbesondere intersektionale Diskriminierung (z. B. Rassismus, Sexismus und Homophobie gegenüber einer lesbischen Frau mit Migrationsbiographie) führen zu einer Vervielfachung des Stresses, da sich im Erleben der Betroffenen die einzelnen Diskriminierungsgründe gegenseitig verstärken (vgl. Carter 2007). Als entscheidende Schutzund Resilienzfaktoren im Kontext von Traumatisierung unterstreicht Gahleitner (2005, S. 63) die Bedeutung von schützenden Inselerfahrungen im Sinne positiver Alternativ-Erfahrungen des Verstehens, der Wertschätzung, Zugehörigkeit und Selbstwirksamkeit für die Betroffenen.

\section{Gesellschaftskritik und globale Verantwortung der Psychotherapie}

Ungleichheits- und Diskriminierungsprozesse sind nicht außerhalb des psychotherapeutischen Raumes verortet. Als Psychotherapeut_innen sind wir „selbst Teil der komplexen Beziehungen und Dynamiken von Differenzproduktion und der Herstellung sozialer Ungleichheit" (Punz 2015, S. 66), sind Privilegierte und teilweise auch Betroffene von Diskriminierung. Die professionelle Beziehungsgestaltung zwischen Therapeut_in und Klient_in weist durch den Fokus auf dem/r Klient_in eine inhärente Macht-Asymmetrie auf. Umso mehr müssen Mechanismen des othering bewusst gemacht und reflektiert werden. Psychotherapiekonzepte der Anerkennung von anderen Kulturen im erweiterten Sinn - sei es bzgl. Aspekten der Transkulturalität, Geschlechtlichkeit, der sexuellen Identität, der körperlichen oder geistigen Befähigung etc. - setzen das Konstruieren der Anderen als anders voraus. „Allein die Frage, wer definiert was als ein Problem, ist kulturell determiniert" (Rommelspacher und Wachendorfer 2008, S. 1337). Das Wissen über Andere kann diese ebenso stereotypisieren wie das Ausblenden von beispielsweise geschlechtlich oder kulturell bzw. rassistisch konstruierten Differenzen. Es ist also gerade die Anerkennung der Differenz, die die Differenz reproduziert und fortschreibt (vgl. Mecheril und Plößer 2018). Insofern geht es weniger darum, als Psychotherapeut_in zur Expert_in für möglichst viele andere Kulturen zu werden, sondern um „die Bereitschaft, die Illusion der Kompetenz zu 
verlieren, d.h. sich von einem instrumentellen $\mathrm{Zu}$ griff auf das ,Wissen über Andere' zu verabschieden“ (Kalpaka und Mecheril 2010, S. 96).

Diversity is not about the others - it is about you (Regine Bendl 2004, S. 56).

Das Differenz-Dilemma (Smykalla 2010, S. 122) ist nicht auflösbar, aber wir können psychotherapeutisch damit arbeiten. Statt die dualistisch gefassten Differenzen zu verstärken, können wir Ambivalenzkompetenz entwickeln und unser „professionelles Handeln als Prozess einer unabgeschlossenen Gleichzeitigkeit“ konzipieren (Smykalla und Vinz 2011, S. 242). Ambivalenzkompetenz in Anlehnung an Butler meint die Fähigkeit, sich auf die Ambivalenz „als eine nicht immer gleich bleibende, aber unvermeidbare Gleichzeitigkeit von Gleichheit und Differenz und von Konstruktion und Dekonstruktion“ einzulassen (ebd.). Auf diese Weise könnte die Psychotherapie „im Dienst einer Vorstellung stehen, die die Menschen in ihren Beziehungen $\mathrm{zu}$ anderen und sich selbst von einer unaufhebbaren Demut charakterisiert sieht. Es gibt immer eine Dimension in uns selbst und in unserer Beziehung zu anderen, von der wir nichts wissen können, und dieses Nicht-Wissen bleibt uns als eine Existenzbedingung und tatsächlich sogar als Überlebensfähigkeit hartnäckig erhalten“ (Butler 2009, S. $30 \mathrm{f}$.).

\section{Ausblick}

Zusammengefasst kristallisieren sich drei Perspektiven heraus, die sich für eine gesellschaftskritische Psychotherapie als hilfreich erweisen (vgl. Rommelspacher und Wachendorfer 2008):

\section{- eine differenzsensible Perspektive}

die vielfältige Lebenslagen (insbesondere soziale, ökonomische und politische Einflussfaktoren), als auch daraus resultierende Bedürfnisse und Erfordernisse von Klient_innen wahrnimmt sowie empathisch versteht und nachempfindet

- eine differenzkritische Perspektive die Prozesse der Anerkennung und gleichzeitigen Reproduktion von Differenz reflektiert und durch die Entwicklung von Ambivalenzkompetenz dazu beiträgt, Normierungen, Stereotypisierungen und Essentialisierungen in der Psychotherapie $\mathrm{zu}$ verringern

\section{- eine dominanzkritische Perspektive}

die othering-Prozesse in- und außerhalb der Psychotherapie dekonstruiert und Differenzen auf ihre Einbettung in die gesellschaftlichen Machtverhältnisse analysiert sowie mit den Positionierungen, insbesondere den Privilegierungen, des/r Psychotherapeut_in transparent umgeht

Das Recht auf Wohlbefinden und ein „erreichbares Höchstmaß an körperlicher und geistiger Gesundheit" ist seit 1966 in einem der mittlerweile zehn UN-
Menschenrechtsabkommen (UN-Sozialpakt 1966, Artikel 12) als grundlegendes Menschenrecht verankert. Die Ethik-Richtlinien des Europäischen Verbandes für Psychotherapie (EAP), die im Jahr 2018 in Belgrad verabschiedet wurden, weisen explizit darauf hin, dass es Aufgabe der Psychotherapie sei, „nach Bewahrung und Schutz der grundlegenden Menschenrechte“ zu streben (EAP 2018). Auch wenn die Idee der Menschenrechte unter einer postkolonial-feministischen Perspektive kritisch hinterfragt und dekolonialisiert werden muss (Varela und Dhawan 2011), so stellen die Menschenrechte dennoch eine unverzichtbare Bezugsgröße für die Psychotherapie dar.

Um den globalen Veränderungen und Herausforderungen angemessen begegnen zu können, muss die Psychotherapie als Menschenrechtsprofession (StaubBernasconi 1995) selbst global werden und ein Selbstverständnis als Profession und wissenschaftlicher Disziplin entwickeln, das psychische Prozesse in einem globalen Kontext versteht (vgl. Sequeira 2015). Vor dem Hintergrund globaler Ungleichheits- und Unterdrückungssysteme (Rassismus, Sexismus, Klassismus etc.) erfährt die westliche Psychotherapie selbst eine Verrückung bzw. De-Zentrierung. Sie ist aufgefordert, ihren Anspruch auf Allgemeingültigkeit in Frage zu stellen und den unsichtbaren Raum, aus dem heraus scheinbar objektiv geforscht und „monologisch die anderen konstruiert wurden“, sichtbar zu machen (Rommelspacher und Wachendorfer 2008, S. 1343). Dieser Perspektivenwechsel hin $\mathrm{zu}$ einer globalen Verantwortung setzt voraus, dass die Psychotherapie Diskurse um Differenz und Intersektionalität aufgreift und die Forschung hinsichtlich gender, queerness, critical whiteness, postcolonialism etc. in ihre Theorie und Praxis einbezieht. Auf diese Weise stellt die Psychotherapie ihr Wirken in den Dienst der SelbstErmächtigung von Menschen in ihrer vielfältigen Verortung von Ungleichheit und Differenz und öffnet kaleidoskopische Räume für neue Beziehungsgestaltungen.

Interessenkonflikt L. Lerch gibt an, dass kein Interessenkonflikt besteht.

Open Access Dieser Artikel wird unter der Creative Commons Namensnennung 4.0 International Lizenz (http:// creativecommons.org/licenses/by/4.0/deed.de) veröffentlicht, welche die Nutzung, Vervielfältigung, Bearbeitung, Verbreitung und Wiedergabe in jeglichem Medium und Format erlaubt, sofern Sie den/die ursprünglichen Autor(en) und die Quelle ordnungsgemäß nennen, einen Link zur Creative Commons Lizenz beifügen und angeben, ob Änderungen vorgenommen wurden.

\section{Literatur}

Baer, S., Bittner, M., \& Göttsche, A.L. (2010). Mehrdimensionale Diskriminierung - Begriffe, Theorien und juristische Analyse. Erstellt im Auftrag der Antidiskriminierungsstelle des Bundes, Berlin, S. 41-45. http://www.antidiskriminierungsstelle.de/SharedDocs/ Downloads/DE/publikationen/Expertisen/Expertise_ 
Mehrdimensionale_Diskriminierung_jur_Analyse.pdf? blob=publicationFile. Zugegriffen:9. Jan. 2019.

Bendl, R. (2004). Gendermanagement und Gender- und Diversitätsmanagement - ein Vergleich der verschiedenen Ansätze.In R.Bendl\&al (Hrsg.), Interdisziplinäres Genderund Diversitymanagement (S.43-73). Wien:Linde.

Böhnisch, L. (2016). Lebensbewältigung. Ein Konzept für die Soziale Arbeit (Zukünfte). Weinheim: BeltzJuventa.

Bryant-Davis, T. \& Ocampo, C. (2005a). Racist incident-based trauma. The Counseling Psychologist, 33, 479-500.

Bryant-Davis, T. \& Ocampo, C. (2005b). The Trauma of Racism: Implicationsfor Counseling, Research andEducation. The Counseling Psychologist, 33, 574-578.

Butler, J. (2001). Psyche der Macht. Das Subjekt der Unterwerfung. Frankfurt a.M.: Suhrkamp.

Butler, J. (2002). Performative Akte und Geschlechterkonstitution. Phänomenologie und feministische Theorie. In U. Wirth (Hrsg.), Performanz. Zwischen Sprachphilosophie und Kulturwissenschaften (S. 301-320). Frankfurt a.M.: Suhrkamp.

Butler, J. (2009). Die Macht der Geschlechternormen und die Grenzen des Menschlichen. Frankfurt a.M.:Suhrkamp.

Butts, H. F. (2002). The black mask of humanity: Racial/ ethnic discrimination and post-traumatic stress disorder. Journal of the American Academy of Psychiatry and the Law, 30(3), 336-339.

Carter, R.T. (2007). Racism and Psychological and Emotional Injury Recognizing and Assessing Race-Based Traumatic Stress. The Counseling Psychologist, 35, 13-105.

Collins, P. H. (1998). Fighting Words. Black Women and the Search for Justice. Minneapolis: University of Minnesota Press.

Comas-Díaz, L. \& Jacobsen, F. M. (2001). Ethnocultural allodynia. The Journal of Psychotherapy Practice and Research, $10,246$.

CombaheeRiverCollective (1981).ABlackFeministStatement. In C. Morage, Anzaldúa G. (Hrsg.), This Bridge Called My Back. Writings by Radical Women of Color (S. 210-218). New York: Women of Color Press.

Davis, A. (1982). Rassismus und Sexismus. Schwarze Frauen und Klassenkampf in den USA. Berlin: Elefanten Press.

Degele, N., \& Winker, G. (2009). Intersektionalität. Zur Analyse sozialer Ungleichheiten. Bielefeld: transcript.

European Association of Psychotherapy (2018). Statement of Ethical Principles. Wien: EAP. https://www.europsyche. org/contents/13134/statement- of- ethical-principles. Zugegriffen: 11.Jan. 2019.

Foucault, M. (1983). Der Wille zum Wissen. Sexualität und Wahrheit I. Frankfurta.M.:Suhrkamp.

Gahleitner, S. B. (2005). Neue Bindungen wagen. Beziehungsorientierte Therapie bei sexueller Traumatisierung. München: Reinhardt.

Gonzalez, M. (2018). Angela Davis and the Distortion of Diversity, Herritage Foundation, 04.05.2018. https://www. heritage.org/civil-society/commentary/angela-davisand-the-distortion-diversity.Zugegriffen: 9. Jan. 2019

Gummich, J. (2004). Schützen die Antidiskriminierungsgesetze vor mehrdimensionaler Diskriminierung? Oder: Von der Notwendigkeit die Ausgeschlossenen einzuschließen (ADEFRA e.V.). In Antidiskriminierungsnetzwerk Berlin des Türkischen Bundes Berlin-Brandenburg (Hrsg.), QUEbERlin. Mehrfachzugehörigkeit als Bürde oder Chance?! Die Gesichter des Queer-Seins \& MigrantIn-/Schwarz-Seins. Tagungsdokumentation, Berlin, 02.12.2004.

Hahn, C., Lerch, L., Reinhard, A., Stein-Banda, R., \&Westen, M. (1999). Möglichkeiten der Grenzerweiterung auf persönli- cher, sozial-politischer und spiritueller Ebene. 22. Feministischer Frauentherapiekongress. Psychosoziale Arbeit im Spannungsfeld unterschiedlicher Kulturen und Lebensformen, 16.-20.06.1999. Berlin: Eigenverlag.

Hall, S. (1994). Rassismus und kulturelle Identität. Ausgewählte Schriften. Hamburg: Argument.

Collins, P. H. (1996). Black women and the sex/gender hierarchy. In S. Jackson \& S. Scott (Hrsg.), Feminism and Sexuality. A reader (S.307-313). New York: Columbia University Press.

Hofmann, R. (2017). Diversitätsgerechte Entwicklungen in Hochschul- und Forschungseinrichtungen. Handreichung für die Praxis. diversitas 2016. Blickpunkte Diversität. Wien: BMWF. https://www.bmbwf.gv.at/fileadmin/ user_upload/gender/2017/Diversitas_Broschuere/ Blickpunkte_Diversitas_WEB_bf.pdf. Zugegriffen: 9. Jan. 2019.

hooks, b. (1981). Ain't I a Woman. Boston, MA: South End Press.

Hull, G. T.; Bell-Scott, P.; Smith, B. (Hrsg.) (1982). All the Women Are White, All the Blacks Are Men, But Some of Us Are Brave. Black Women's Studies. NewYork:The Feminist Press.

Igel, U., Brähler, E., \& Grande, G. (2010). Der Einfluss von Diskriminierungserfahrungen auf die Gesundheit von MigrantInnen. Psychiatrische Praxis, 37(4), 183-190.

Internationaler Pakt über bürgerliche und politische Rechte (UN-Sozialpakt) (1966). Praetor Intermedia UG, Bonn. https://www.sozialpakt.info/internationalerpakt-ueber-wirtschaftliche-soziale-und-kulturellerechte-3111.Zugegriffen: 11.Jan. 2019.

Kalpaka, A., \& Mecheril, P. (2010). Interkulturell'. Von spezifisch kulturalistischen Ansätzen zu allgemein reflexiven Perspektiven. In P. Mecheril, et al. (Hrsg.), Migrationspädagogik (S.77-98). Weinheim: Beltz.

Keilson, H. (1979). Sequentielle Traumatisierung bei Kindern. Stuttgart:Enke.

Kerchner, B. (2011). Diskursanalyse der Intersektionalität. In S. Smykalla \& D. Vinz (Hrsg.), Intersektionalität zwischen Gender und Diversity. Theorien, Methoden und Politiken der Chancengleichheit. Schriftreihe der Sektion Frauen und Geschlechterforschung in der Deutschen Gesellschaft für Soziologie, (Bd. 30, S. 144-161). Münster: Westfälisches Dampfboot.

Keupp, H., \& Bilden, H. (Hrsg.). (1989). Verunsicherungen. Das Subjekt im gesellschaftlichen Wandel. Göttingen: Verlag für Psychologie.

Khan, M.M.R. (1963). Das kumulative Trauma. In M. M.R. Khan (Hrsg.), Selbsterfahrung in der Therapie (S. 50-70). München:Kindler.

Kilomba, G. (2008). Plantation memories. Stories of everyday racism (S.94-100). Münster: Unrast.

Klaus, E. (2005). Kommunikationswissenschaftliche Geschlechterforschung. Zur Bedeutung der Frauen in den Massenmedien und im Journalismus. Medien- und Geschlechterforschung, Bd.7. Münster:LIT.

Klinger, C., \& Knapp, G.-A. (2007). Achsen der Ungleichheit Achsen der Differenz. Verhältnisbestimmung von Klasse, Geschlecht und Ethnizität. In C. Klinger \& G.-A. Knapp (Hrsg.), Achsen der Ungleichheit - Achsen der Differenz. Verhältnisbestimmung von Klasse, Geschlecht und Ethnizität(S. 19-41). Frankfurta.M.: Campus.

Lerch, L. (2011). Rassismus. Auswirkungen auf die psychische Gesundheit. In Wiener Landesverband für Psychotherapie (Hrsg.), WLP-News 2/2011 (S. 9-13). Wien: Wiener Landesverband für Psychotherapie.

Lerch, L. (1997a). Feminismen - Rassismen. Dialog zwischen Schwarzen und Weißen Feministinnen über die 
Möglichkeit und Unmöglichkeit von Bündnissen. In A. Blessing, et al. (Hrsg.), 20 Jahre Feministische Beratung, Therapie, Supervision. Entwicklung, Bilanz, Perspektiven (S. 74-81). München: Frauentherapiezentrum München e. V..

Loo, C. M., Fairbank, J. A., Scurfield, R. M., Ruch, L. O., King, D. W., Adams, L. J. \& Chemtob, C. M. (2001). Measuring exposure to racism: Development and validation of a Race-Related Stressor Scale (RRSS) for Asian American Vietnam veterans. Psychological Assessment, 13, 503.

Lorde, A. (1988). Age, Race, Class, and Sex. Women Redefining Difference. In P. S. Rothenberg (Hrsg), Racism and Sexism. An Integrated Study (S. 352-359). New York: St. Martin's Press.

Lorde, A. (1994).Zami. Ein Leben unter Frauen. Frankfurta.M.: Fischer.

Mahler, L., Mundle, G., \& Plöderl, M. (2018). Wirkungen und Nebenwirkungen des Krankheitskonzepts, Homosexualität'. Fortschritte Neurologie, Psychiatrie, 86, 469-476.

McCall, L. (2001). Complex Inequality. Gender, Class and Race in the New Economy. New York: Routledge.

Mecheril, P., \&Plößer, M. (2018). Diversityund SozialeArbeit. In H.-U. Otto \& al (Hrsg.), Handbuch Soziale Arbeit (6. Aufl. S. 283-292). München: Reinhardt.

Meyer, I. (2003). Prejudice, social stress, and mental health in lesbian, gay, and bisexual populations. Conceptual issues and research evidence. Psychological Bulletin, 129, 674-697.

Paradies, Y. C. \& Cunningham, J. (2012). The DRUID study: Exploring mediating pathways between racism and depressive symptoms among Indigenous Australians. Social Psychiatry and Psychiatric Epidemiology, 47, 165-173.

Punz, J. (2015). Perspektiven intersektional orientierter Sozialer Arbeit. Dimensionen des Umgangs mit Differenzkonstruktion und Diskriminierung in der Praxis Sozialer Arbeit. Soziales Kapital - Wissenschaftliches Journal Österreichischer Fachhochschul-Studiengänge Soziale Arbeit, 13, 63-74. http://sozialeskapital.at/index. $\mathrm{php} /$ sozialeskapital/article/viewFile/365/611.pdf. Zugegriffen: 7. Jan. 2019

Rommelspacher, B. (1995). Dominanzkultur. Texte zu Fremdheit und Macht (1.Aufl.). Berlin: Orlanda.

Rommelspacher, B., \&Wachendorfer, U. (2008). Interkulturelle Therapie. In M. Hermer \& B. Röhrle (Hrsg.), Handbuch der therapeutischen Beziehung (Bd. 2, S. 1337-1360). Tübingen: dgvt.

Scheffler, S. (2010). und sie bewegt sich doch! Entwicklung und Zukunft der frauenspezifischen Psychotherapie und Beratung. In Frauen beraten Frauen (Hrsg.), In Anerkennung der Differenz. Feministische Beratung und Psychotherapie(S. 45-57). Gießen:Psychosozial-Verlag.
Sequeira, D. (2015). Gefangen in der Gesellschaft - Alltagsrassismus in Deutschland. Rassismuskritisches Denken und Handeln in der Psychologie. Marburg: Tectum.

Smykalla, S. (2010). Die Bildung der Differenz. Weiterbildung und Beratung im Kontext von Gender Mainstreaming. Wiesbaden:VS.

Smykalla, S., \& Vinz, D. (2011). Geschlechterforschung und Gleichstellungspolitiken vor neuen theoretischen, methodologischen und politischen Herausforderungen. In ebd (Hrsg.), Intersektionalität zwischen Gender und Diversity. Theorien, Methoden und Politiken der Chancengleichheit.SchriftreihederSektionFrauenundGeschlechterforschungin der Deutschen Gesellschaftfür Soziologie, Bd.30. Münster:Westfälisches Dampfboot.

Spivak, \& Chakravorty, G. (1985). The rani of Sirmur. An essay in reading the archives. History and Theory, 24(3), 247-272.

Staub-Bernasconi, S. (1995). Das fachliche Selbstverständnis Sozialer Arbeit - Wege aus der Bescheidenheit. Soziale Arbeit als „Human Rights Profession“. In W. R. Wendt (Hrsg.), Soziale Arbeit im Wandel ihres Selbstverständnisses: Beruf und Identität. Schriftenreiheder Deutschen Gesellschaft für Sozialarbeit e. V., (Bd. 2, S. 57-104). Freiburg i.B: Lambertus.

Varela, M.d.M. C., \&Dhawan, N. (2011). Soziale (Un)Gerechtigkeit. Kritische Perspektiven auf Diversity, Intersektionalität und Antidiskriminierung. Politikwissenschaft, Bd. 158. Berlin:LIT.

Walgenbach, K., Dietze, G., Hornscheidt, A., \& Palm, K. (2012). Gender als interdependente Kategorie. Neue Perspektiven auf Intersektionalität, Diversität und Heterogenität(2.Aufl.).Leverkusen: Budrich.

West, C., \& Fenstermaker, S. (1995). Doing Difference. Gender and Society, 9(1), 8-37.

West, C., \& Zimmerman, D. (1987). Doing Gender. Gender and Society, 1(2), 125-151.

Wolf, C. (1983). Voraussetzungen einer Erzählung: Kassandra. Darmstadt:Luchterhand.

Yeboah, A. (2015). Rassismus und psychische Gesundheit in Deutschland. Hintergrundpapier zum Parallelbericht an den UNAntirassismusausschuss zum 19.-22. Bericht der Bundesrepublik Deutschland nach Artikel 9 des Internationalen Übereinkommens zur Beseitigung jeder Form von rassistischer Diskriminierung. Berlin: Forum Menschenrechte e.V. https://rassismusbericht. de/wp-content/uploads/Rassismus-und-psychischeGesundheit.pdf.Zugegriffen:11.Jan.2019.

Hinweis des Verlags Der Verlag bleibt in Hinblick auf geografische Zuordnungen und Gebietsbezeichnungen in veröffentlichten Karten und Institutsadressen neutral. 